\title{
Pleural empyema in children - benefits of primary thoracoscopic treatment
}

\author{
Roksana Barglik, Andrzej Grabowski, Wojciech Korlacki, Michał Pasierbek, Anna Modrzyk \\ Department of Children Developmental Defects Surgery and Traumatology in Zabrze, School of Medicine with the Division of Dentistry \\ in Zabrze, Medical University of Silesia in Katowice, Poland
}

Videosurgery Miniinv 2021; 16 (1): 264-272

DOI: https://doi.org/10.5114/witm.2020.97443

\begin{abstract}
Introduction: Pleural empyema is the condition of the pleural cavity when initially sterile pleural effusion has become infected. In the majority of cases, it is of parapneumonic origin. Parapneumonic effusions and pleural empyemata usually continuously progress in severity. The American Thoracic Society divides them into three stages: exudative, fibrinopurulent and organizing. The therapy depends on the stage.

Aim: To assess whether thoracoscopy should be considered better than conservative treatment and to assess the feasibility of the thoracoscopic approach to the $3^{\text {rd }}$ phase of pleural empyema.

Material and methods: The clinical course of 115 patients treated from 1996 to 2017 was analyzed. 45 patients operated on thoracoscopically after the failure of conventional treatment were compared with 70 patients treated by primary thoracoscopic drainage and decortication.

Results: The results of the study demonstrated that patients treated primarily by thoracoscopy had a shortened length of hospital stay (16.6 vs. 19.3 days), reduced drainage time (7.9 vs. 9.8 days), and shorter time of general therapy (31.8 vs. 38.0 days). They required fibrinolysis less frequently (12.8 vs. $26.7 \%$ of patients) and had reduced risk of reoperation (10 vs. $15.6 \%$ of cases). Operation time in the $3^{\text {rd }}$ stage was only 15 min longer. The difference in length of hospital stay was only 0.8 days in favor of less severe cases.

Conclusions: The thoracoscopic approach is safely feasible in the $3^{\text {rd }}$ stage of pleural empyema and should be considered as the preferred approach. Furthermore, the post-operative stay and general course of the disease are milder whenever surgery would not be delayed by prolonged conservative treatment attempts.
\end{abstract}

Key words: video-assisted thoracic surgery, management, child, pleural empyema, thoracoscopy, empyema thoracis.

\section{Introduction}

Pleural empyema (PE) is the condition of the pleural cavity when initially sterile pleural fluid has become infected. It is observed in $10 \%$ of cases of community acquired pneumonia, which in children constitutes about 3.3 per $100000[1,2]$. The collection becomes pus when the concentration of leucocytes starts to be macroscopically evident as a dense and turbid fluid. In the majority of cases, it is of parapneumonic origin. Other not so common causes include surgical procedures (mainly thoracic surgery), traumas and esophageal perforation, but these are rare in children. Conditions are more common in boys than girls and are more frequently encountered in infants and young children. They are also more common in winter and spring, presumably due to their infective origin $[2,3]$. It has been suggested that the incidence of childhood empyema is increasing, although this is not a universal find-

\section{Address for correspondence}

Roksana Barglik, Department of Children Developmental Defects Surgery and Traumatology, School of Medicine with the Division of Dentistry, Medical University of Silesia, 3 maja 13-15, 41-800 Zabrze, Poland, phone: +48 780 050 600, e-mail: roxi01@interia.pl 
ing. Parapneumonic effusions and empyema usually progress in severity continuously, which led to them being divided into three stages: exudative, fibrinopurulent and organizing, commonly named $1^{\text {st }}, 2^{\text {nd }}$ and $3^{\text {rd }}$ phase according to the American Thoracic Society $[3,4]$. Patients can progress to another stage within hours if effective treatment is not provided [1]. The therapy is stage dependent and since Hippocrates' times the evacuation of pus has been considered essential. Additionally, in the third phase the organized, thick fibrous peel in the pleural cavity restricts lung expansion, which causes chronic sepsis and intensifies the respiratory insufficiency. That is called "trapped lung" and requires excision of the pleural rinds to achieve proper lung expansion and ventilation [2]. The procedure is called decortication and conventionally it was done through proper thoracotomy. Only a small amount of experience has been published about decortication in the late phase of pleural empyema via a minimally invasive thoracoscopic approach.

\section{Aim}

It is prospective non-randomized cohort study. Patient data were retrospectively analyzed from the prospectively maintained institutional database. We set two goals for the study.

The first one was to assess whether the early surgical intervention should be considered better than conservative treatment with the insertion of a chest tube or thoracentesis. The primary end point was the length of hospitalization and therapy of patients referred to our center with and those without prior interventional therapy.

The second aim was to assess the feasibility, safeness and radicality of the thoracoscopic approach to the organizational, $3^{\text {rd }}$ phase of pleural empyema. Again, the end point was the length of hospitalization but also the number of complications and adverse events in patients in the $3^{\text {rd }}$ phase compared to those in less severe stages.

In both groups, the length of hospitalization was limited by criteria assessed according to Melloni, which take into account elimination of symptoms, normalization of white blood cells (WBC) count, removal of drain, return to normal (for the patient) physical activity and favorable X-ray image of the chest [5].

The study was approved by the local ethics committee of the medical faculty.

\section{Material and methods}

Qualification for thoracoscopic intervention was performed in the pediatric-surgical team based on the history, clinical symptoms and imaging tests according to the established pattern.

Indications for referral to the thoracoscopic treatment were:

- intensification of the disease process of the lung tissue with the involvement of at least one pleural cavity,

- confirmation of the presence of fluid and/or fibrous layers forming the loculations in the pleural cavity in radiological and ultrasound examinations of the chest,

- symptoms in physical examination and laboratory tests indicating an infectious cause of fluid in the pleural cavity (fever, cough, poor general condition, increase in inflammatory parameters).

\section{Technique of the procedure}

The thoracoscopy procedure was performed under general anesthesia with endotracheal intubation with a single lumen endotracheal tube. The patient was placed in a lateral decubitus position with pronounced intercostal space. The place of insertion of the first $5 \mathrm{~mm}$ or $10 \mathrm{~mm}$ optical trocar was intercostal space V-VIII (usually VI or VII) in the anterior or medial axillary line. The $5 \mathrm{~mm}$ working trocars (one or two) were located under optic control and depending on the anatomical conditions prevailing in the inflamed pleural cavity. First, exudative or purulent content was evacuated and material for bacteriological examination was collected. In the case of the presence of adhesions, they were released to combine the individual empyema loculations to obtain one pleural space, which allowed evacuation of the remaining purulent content.

In the case of stage III of the empyema with the presence of thick fibrous layers on the parietal and pulmonary pleura, they were dissected laterodorsally and towards the mediastinum by peeling the lung's visceral pleura of the fibrous cortex encapsulating the empyema and all the thickened parietal pleura was decorticated completely. This was performed until the lung was mobilized completely and the hilum was reached. Particular attention was paid to release the pulmonary ligament. In addition, the fissures were debrided as much as possible to achieve proper lung expansion. The peeled fibrous 
fragments were removed from the thoracic cavity though the trocar or if they were too thick straight through the trocar site wound with temporary trocar withdrawal. In the next step, a specimen was taken from the pleural wall for histopathological examination. The last stage of the procedure was placement of a drain in strategic locations in the pleural cavity under direct vision.

Criteria for inclusion:

- patients who underwent thoracoscopy due to pleural empyema in the years 1996 to 2017,

- patients with full medical documentation regarding the course of the disease,

- patients with full medical documentation regarding thoracoscopic surgery.

Exclusion criteria:

- patients with other than inflammatory background of fluid in the pleural cavity (chylothorax, exudation in neoplastic diseases, haemothorax),

- patients with incomplete medical documentation regarding the course of the disease,

- patients with incomplete medical documentation regarding thoracoscopic surgery.

On the basis of the above-mentioned medical documentation, demographic data (gender, age), basic symptoms and their duration, current treatment, co-morbidity, clinical stage of the process, its location, procedures performed during the operation,
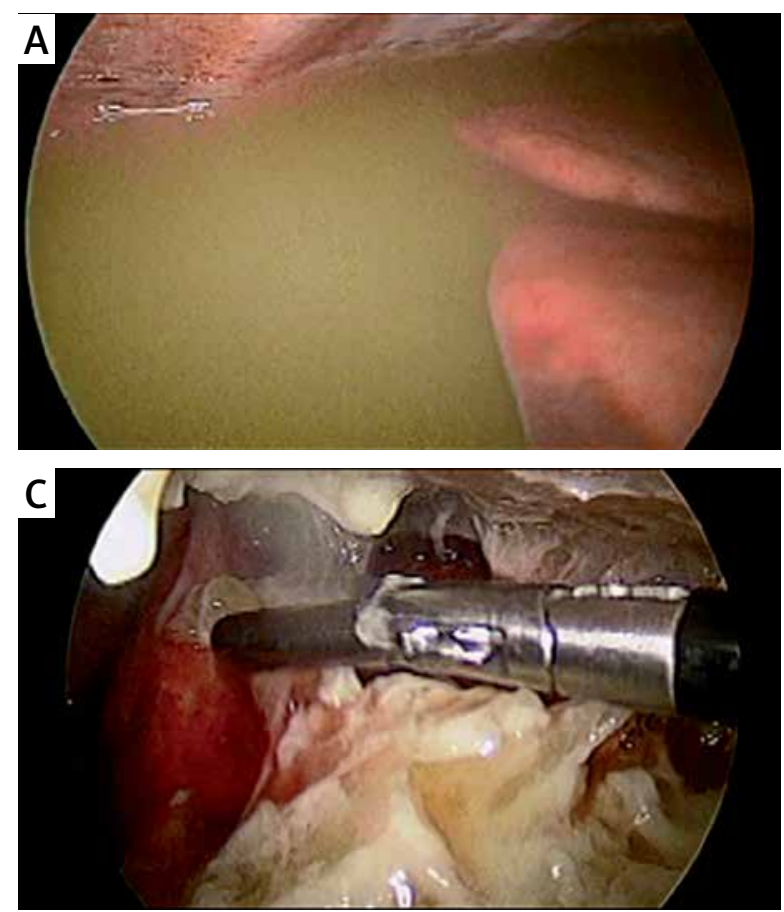

its duration, presence of other pathologies found during the procedure, intra-operative complications, post-operative adverse events, including the necessity for re-intervention, postoperative period, further treatment, duration of the drainage, and length of hospitalization were evaluated.

Patients were divided into two groups for the first goal evaluation: PD group - patients undergoing puncture and/or drainage of the pleural cavity before transfer to our department and implementation of surgical, thoracoscopic treatment according to our protocol; T group - patients primarily treated by thoracoscopy.

To assess the second goal, we followed the American Thoracic Society classification of clinical stages (phases): $1^{\text {st }}$ exudative, $2^{\text {nd }}$ fibrinopurulent (together constituted the first group) and $3^{\text {rd }}$, organizing phase (the second group). The assessment was made according to radiological imaging (radiography, ultrasound imaging, computed tomography) not determined by symptom duration and further reassessment was made after the procedure. In the following results only, those confirmed in such manner were included in the proper stage (Photo 1).

\section{Statistical analysis}

The obtained results were subjected to statistical analysis. Statistica 12 was used in statistical anal-

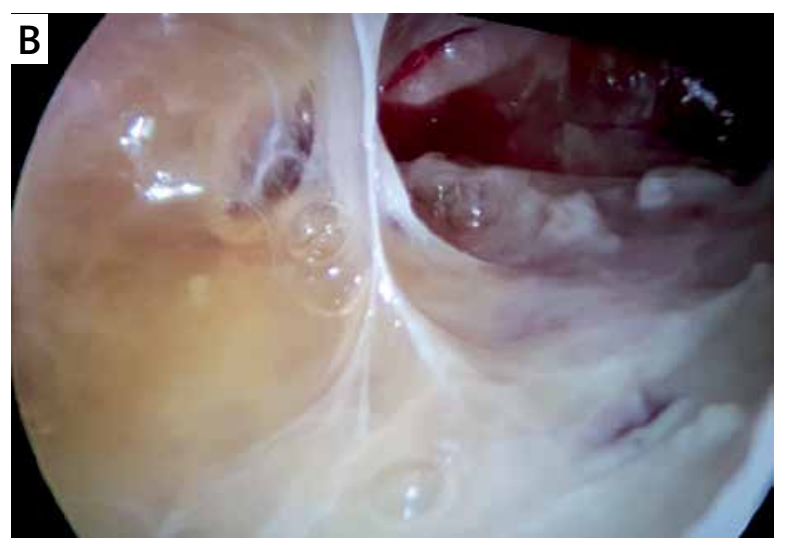

Photo 1. Intraoperative images: $\mathrm{A}$ - empyema in $1^{\text {st }}$ stage, $\mathbf{B}$ - empyema in $2^{\text {nd }}$ stage, $\mathbf{C}$ - empyema in $3^{\text {rd }}$ stage 
ysis of results. Normality of data distribution was checked by the Kolmogorov-Smirnov test. In order to compare continuous variables in the studied groups, Student's $t$-test was used for data with normal distribution, and the results were presented as the mean value and standard deviation. For non-normal distribution data, the Mann-Whitney $U$ test was used, and the results were presented as the median, minimum and maximum values. The distribution of dichotomous variables was compared with the $\chi^{2}$ test and the Fisher exact test was used for small numbers. $P<0.05$ was considered statistically significant.

\section{Results}

In the period from 1996 to 2017, 115 surgical interventions were performed in children with pleural empyema in our department. Among those treated were 50 (43.48\%) girls and 65 (56.52\%) boys from 12 months to 18 years (mean 7 years, median 5 years) (Figure 1).

During the study period we found an increase both in total incidence of empyema and its severity counted as the number of patients in the third clinical phase (Figure 2).

Forty-five patients were referred to our center after initial interventional treatment (thoracentesis - 30, tube insertion - 24, numbers overlapped) after mean time of 18.7 days of therapy elsewhere. 70 were admitted without prior treatment despite antibiotics; for them average time of therapy prior to admission was 15.4 days.

The location of empyema occurred with a similar frequency in both pleural cavities $-51.3 \%$ were lo-

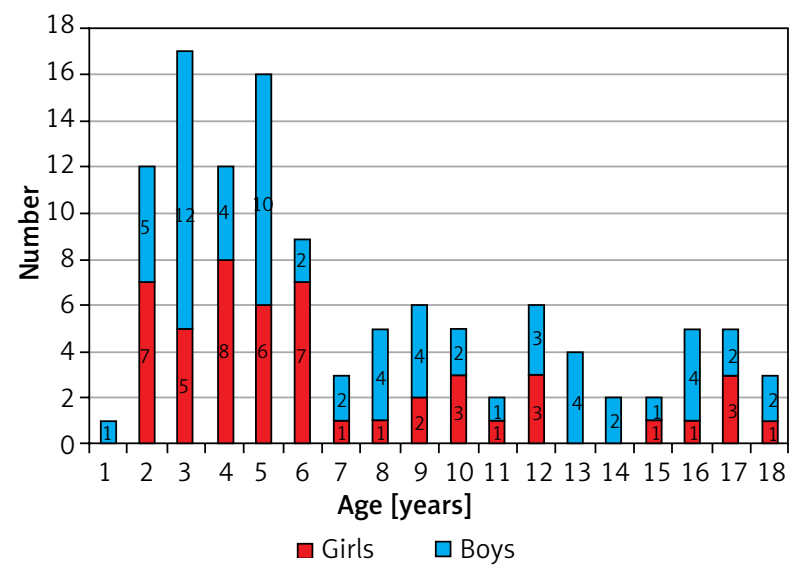

Figure 1. Age and sex distribution of pleural empyema in children. The peak of incidence is in the 2- to 6-year-old age range cated in the right one and $48.7 \%$ in the left pleural cavity. In both groups, the frequency of location of lesions on the right and left were similar as it was according to severity of the lesion.

Intraoperatively, the severity of the empyema, the accumulation of fibrinous aggregates forming the empyema chamber, the coexistence of the bronchopleural fistula or any other pathologies, among which the most frequent is pulmonary parenchymal destruction, presence of lesions suggesting tuberculous background, and coexistence of pulmonary abscesses or emphysema blebs, were assessed.

The stage of pleural empyema was determined depending on the nature of the effusion, the presence of septations and loculations or fibrous adhesions of the parietal and pulmonary pleura found during thoracoscopy.

The number of cases depending on the stage of empyema was: 8 for phase 1, 46 for phase 2, 61 for phase 3 (Table I). All those in the third phase were treated with decortications.

Mean operating time was 52 min (range: 15-120). For the patients in the $1^{\text {st }}$ and $2^{\text {nd }}$ phase it was $44 \mathrm{~min}$ on average and for those in the $3^{\text {rd }}$ stage it was 59 min.

There were no conversions. There was one intraoperative, procedure-dependent complication - an improperly placed trocar perforated through the costodiaphragmatic recess into the peritoneal cavity and injured the liver. Urgent laparotomy solved the problem. The other important features found were

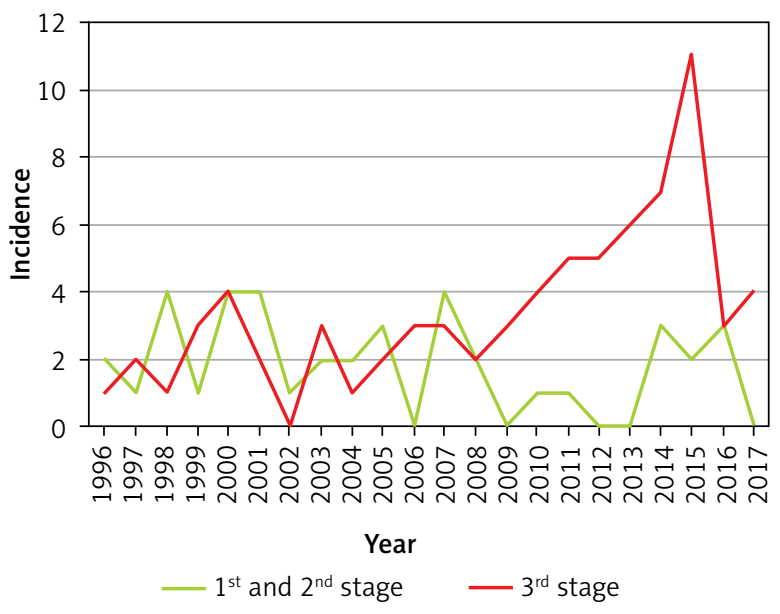

Figure 2. Incidence of pleural empyema in children during the period 1996-2017. The figure represents the number of diagnosed cases per year 
Table I. The number of cases depending on the stage of empyema

\begin{tabular}{|lccc|}
\hline \multicolumn{2}{|l|}{ The number of cases depending on the stage of empyema } & In PD group & In T group \\
\hline $1^{\text {st }}$ (exudative) & $8(7 \%)$ & $1(2.2 \%)$ & $7(10.0 \%)$ \\
\hline $2^{\text {nd }}$ (fibrinopurulent) & $46(40 \%)$ & $19(42.2 \%)$ & $27(38.6 \%)$ \\
\hline $3^{\text {rd }}$ (organizing) & $61(53 \%)$ & $25(55.6 \%)$ & $36(51.4 \%)$ \\
\hline Total & 115 & 45 & 70 \\
\hline
\end{tabular}

almost exclusively in the third phase patients: there were 4 bronchopulmonary fistulas, 5 lung abscesses, 4 necroses of lung tissue. In 1 case the patient was admitted with the chest tube inserted through the left costodiaphragmatic recess into the peritoneal cavity, which caused a small perisplenic abscess. During the thoracoscopic procedure the ruptured diaphragm was sutured.
Reoperations were performed in 13 (11.3\%) patients - 7 (15.6\%) from the PD group and 6 (8.6\%) from the $\mathrm{T}$ group. Patients who required reoperations were exclusively in third phase empyema. All of them also had additional pathologies mentioned above. All reoperations were performed thoracoscopically. Moreover, in 21 patient a fibrinolytic agent was poured through the drain into the pleural

Table II. Comparison of clinical characteristics $-1^{\text {st }}$ and $2^{\text {nd }} v s .3^{\text {rd }}$ stage of empyema

\begin{tabular}{|c|c|c|c|}
\hline Variables & Stage I + II $(n=54)$ & Stage III $(n=61)$ & Total $(n=115)$ \\
\hline Age [years] & $7.6 \pm 4.3$ & $6.6 \pm 5.3$ & $7.4 \pm 4.9$ \\
\hline Gender: female & $24(44.4 \%)$ & $26(42.6 \%)$ & $50(43.5 \%)$ \\
\hline Right side lesions & $26(48.1 \%)$ & $33(54.1 \%)$ & $59(51.3 \%)$ \\
\hline Fever & $53(98.1 \%)$ & $57(93.4 \%)$ & $110(96.5 \%)$ \\
\hline Cough & $45(83.3 \%)$ & $43(70.5 \%)$ & $88(77.2 \%)$ \\
\hline Chest pain & $25(46.3 \%)$ & $40(65.6 \%)$ & $59(51.8 \%)$ \\
\hline Dyspnoea & $25(46.3 \%)$ & $34(55.7 \%)$ & $65(57.0 \%)$ \\
\hline Leucocyte count [g/l] & $14.9 \pm 7.5$ & $17.7 \pm 8.9$ & $16.4 \pm 8.4$ \\
\hline Neutrophils (\%) & $74.3 \pm 13.0$ & $73.4 \pm 13.7$ & $73.9 \pm 13.3$ \\
\hline $\mathrm{CRP}[\mathrm{mg} / \mathrm{l}]$ & $153.9 \pm 133.1$ & $147.6 \pm 98.8$ & $150.5 \pm 114.6$ \\
\hline $\mathrm{ESR}[\mathrm{mm} / \mathrm{h}]$ & $91.2 \pm 41.5$ & $94.3 \pm 42.8$ & $92.7 \pm 41.9$ \\
\hline Bronchopulmonary fistulas & $2(3.7 \%)$ & $8(13.1 \%)$ & $10(8.7 \%)$ \\
\hline Lung abscesses & $0(0 \%)$ & $4(6.5 \%)$ & $4(3.5 \%)$ \\
\hline Necrosis of lung tissue & $0(0 \%)$ & $4(6.5 \%)$ & $4(3.5 \%)$ \\
\hline Tuberculosis & $6(11.1 \%)$ & $3(4.9 \%)$ & $9(7.8 \%)$ \\
\hline Duration of complaint [days] & $13.7 \pm 8.4$ & $19.4 \pm 10.8$ & $17.6 \pm 10.9$ \\
\hline Mean operating time [min] & $44.3 \pm 15.4$ & $59.4 \pm 21.0$ & $52.3 \pm 20.0$ \\
\hline Fibrinolysis & $10(18.5 \%)$ & $11(18.0 \%)$ & $21(18.3 \%)$ \\
\hline Reoperations & $3(5.6 \%)$ & $11(18.0 \%)$ & $14(12.2 \%)$ \\
\hline Length of hospital stay & $17.3 \pm 12.3$ & $18.0 \pm 12.3$ & $17.7 \pm 12.2$ \\
\hline Time of drainage & $7.8 \pm 4.7$ & $9.3 \pm 6.6$ & $8.6 \pm 5.8$ \\
\hline General time of therapy & $31.0 \pm 13.8$ & $37.1 \pm 26.6$ & $32.8 \pm 15.4$ \\
\hline
\end{tabular}


cavity. In all those patients, dilution of thick, drain obstructing pus was achieved. 10 of those patients came from the $2^{\text {nd }}$ phase group, 11 from the $3^{\text {rd }}$.

Mean length of hospital stay after the thoracoscopic procedure was 17.7 days (range: 4-59). It was 19.3 days for the PD group and 16.6 for group T. It was also 17.2 days for patients in the $1^{\text {st }}$ and $2^{\text {nd }}$ phase and 18.0 days for patients in the $3^{\text {rd }}$ phase (Table II).

In 9 cases tuberculosis was diagnosed based on histopathological examination of the pleural specimen. Bacteriology was positive in 13 cases only; in this patients targeted antibiotic therapy was administered. Otherwise empiric antibiotic therapy such as cephalosporins ( $2^{\text {nd }}$ and $3^{\text {rd }}$ generation), penicillins and penicillin combinations, macrolides, quinolones, carbapenems, glycopeptides, oxazolidinones and less frequently aminoglycosides, lincosamides and antifungals was given. Mean time of drainage of the pleural cavity after the procedure was 8.6 days. In $1^{\text {st }}$ and $2^{\text {nd }}$ phases it was 7.8 days, in the $3^{\text {rd }}$ phase 9.3 days (Table III).

There were no deaths during the hospital stay or during follow-up. Mean general time of therapy was 34 days (range: 11-197) with no significance in PD or T groups or $1^{\text {st }} / 2^{\text {nd }} v$ v. $3^{\text {rd }}$ phase.

\section{Discussion}

First goal: The length of hospital stay between groups with or without procedures prior to thoracoscopy differed in 3 days only, which was statistically significant. Adding the time of therapy before admis-

Table III Relation between type of management and clinical outcome

\begin{tabular}{|c|c|c|c|}
\hline Variables & PD group $(n=45)$ & T group $(n=70)$ & Total $(n=115)$ \\
\hline Age [years] & $6.7 \pm 5.0$ & $7.6 \pm 4.6$ & $7.4 \pm 4.9$ \\
\hline Gender: female & $16(35.6 \%)$ & $34(48.6 \%)$ & $50(43.5 \%)$ \\
\hline Right side lesions & $25(55.6 \%)$ & $34(48.6 \%)$ & 59 (51.3\%) \\
\hline Fever & $43(95.6 \%)$ & $67(95.7 \%)$ & $110(96.5 \%)$ \\
\hline Cough & $35(77.8 \%)$ & $53(75.7 \%)$ & $88(77.2 \%)$ \\
\hline Chest pain & $30(66.7 \%)$ & $35(50 \%)$ & $59(51.8 \%)$ \\
\hline Dyspnoea & $24(53.3 \%)$ & $35(50 \%)$ & $65(57.0 \%)$ \\
\hline Leucocyte count [g/l] & $15.3 \pm 6.3$ & $17.0 \pm 9.4$ & $16.4 \pm 8.4$ \\
\hline Neutrophils (\%) & $75.3 \pm 8.9$ & $72.9 \pm 15.5$ & $73.9 \pm 13.3$ \\
\hline CRP $[\mathrm{mg} / \mathrm{l}]$ & $151.2 \pm 122.1$ & $149.9 \pm 111.4$ & $150.5 \pm 114.6$ \\
\hline $\mathrm{ESR}[\mathrm{mm} / \mathrm{h}]$ & $90.5 \pm 49.6$ & $94.5 \pm 34.5$ & $92.7 \pm 41.9$ \\
\hline Bronchopulmonary fistulas & $5(11.1 \%)$ & $5(7.1 \%)$ & $10(8.7 \%)$ \\
\hline Lung abscesses & $1(2.2 \%)$ & $3(4.3 \%)$ & $4(3.5 \%)$ \\
\hline Necrosis of lung tissue & $1(2.2 \%)$ & $3(4.3 \%)$ & $4(3.5 \%)$ \\
\hline Tuberculosis & $2(4.4 \%)$ & $7(10 \%)$ & $9(7.8 \%)$ \\
\hline Duration of complaint [days] & $18.7 \pm 9.0$ & $15.4 \pm 10.3$ & $17.7 \pm 10.9$ \\
\hline Mean operating time [min] & $51.3 \pm 18.9$ & $52.9 \pm 20.8$ & $52.3 \pm 20.0$ \\
\hline Fibrinolysis & $12(26.7 \%)$ & $9(12.8 \%)$ & $21(18.3 \%)$ \\
\hline Reoperations & $7(15.6 \%)$ & $7(10.0 \%)$ & $14(12.2 \%)$ \\
\hline Length of hospital stay & $19.3 \pm 12.4$ & $16.6 \pm 12.1$ & $17.7 \pm 12.2$ \\
\hline Time of drainage & $9.8 \pm 6.1$ & $7.9 \pm 5.6$ & $8.6 \pm 5.8$ \\
\hline General time of therapy & $38.0 \pm 29.0$ & $31.8 \pm 14.9$ & $32.8 \pm 15.4$ \\
\hline
\end{tabular}


sion to our center the difference is another 6 days longer, but we cannot discuss the criteria for thoracentesis, drainage or prevailing pharmacology in other centers. Thus, we were not able to reveal any advantage or disadvantage of conducting any procedures before thoracoscopy. In multicenter studies from Brazil they found a decrease of postoperative hospital length of stay in patients referred straight for thoracoscopy comparing those with initial chest tube placement and then thoracoscopy, but overall time to resolution was similar in both groups. It supports the suggestion that tube placement was not curative at all [6]. Even the bacteriology sample was usually useless as most of the examinations were sterile, both in our material and the literature [7].

Moreover, the increasing number of phase $3 \mathrm{em}$ pyemata seems to be an epidemiological trend, not an effect of clinical decisions [8]. This cannot help in ongoing discussion in favor of early or late surgery. Thus, in decision making other indications should be taken into consideration. So, following the guidelines we claim that, if the child is undergoing general anesthesia for simple drain insertion anyway, the procedure should be combined with video-assisted thoracic surgery (VATS) [2].

Second goal: The length of post-operative hospital stay was used as the primary endpoint for measuring patient outcome after surgery. Multiple regression analysis focused on this same parameter as it is directly influenced by postoperative period and thus by chest tube duration, reoperation rate and post-operative complications.

We found no statistically significant differences in postoperative course between patients in the $1^{\text {st }}$ and $2^{\text {nd }}$ (counted together) vs. the $3^{\text {rd }}$ phase of empyema. The time of procedures was longer and the numbers of perioperative findings and reoperations were higher in patients in the organizational phase, but it was easy to predict. But despite that fact, mean length of hospital stay and overall time of therapy were not statistically significantly longer than for those in the $1^{\text {st }}$ and $2^{\text {nd }}$ phase.

Complete removal of the fibrotic stratifications trapping the lung is of vital importance for the success of surgical therapy in the $3^{\text {rd }}$ phase. Any remaining cortex on the lung's surface preventing its complete re-expansion leads to recurrent pleural effusion, which involves a high risk for getting re-infected, mainly when the underlying pneumonia is not fully recovered at the time of surgery. Entrapping the lung also worsens the perfusion of the lung, which makes the tissue less prone to the curative effect of antibiotics $[2,4]$. Therefore, the feasibility and radicality of the use of thoracoscopy in phase 3 empyema are not widely recommended in favor of proper thoracotomy $[2,7]$. The benefits of a minimally invasive approach, being less postoperative pain, quicker recovery and less chance for scoliosis, are well known, so it makes thoracoscopy for empyema worth doing as long it is safe enough [9-11]. However, we believe that with the presented material we proved that thoracoscopy is safe enough and feasible enough to replace the thoracotomy in most cases.

Concerning the safeness, the sole major complication - inserting the trocar into the peritoneal cavity - happened in the patient with cerebral palsy and major deformations of the thorax, thus being prone to greater risk in the open approach as well. On the other hand, we had admitted from the other hospital one quite properly developed patient in whom a chest tube was inserted into the abdomen through the costodiaphragmatic recess.

Concerning limits of radicality, one can always switch VATS to thoracotomy with no additional harm for the patient than if starting with thoracotomy. This is suggested as more reliable in terms of predictors of a successful operation [12]. But with patience and ordinary equipment decortication is feasible via thoracoscopy as most of the fibrous stratifications are prone to blunt dissection and they are not firmly connected to lung tissue, so it is possible to enter the cavity even if it seems to be completely overgrown.

Similar findings were presented from adult cohorts [4, 13-15]. Thus, the recent expert consensus statement the European Association for Cardio-Thoracic Surgery (EACTS) has recommended a primary VATS approach for the surgical management even for the organized phase [16].

We do not consider pharmacological fibrinolysis as equivalent or superior to surgery. The fibrinolytic agents are not approved for empyema therapy in our country and thus we avoid using them in first line therapy. Opinions about effects and mechanism of fibrinolysis are still debatable and both the effect and mechanism of fibrinolysis are questioned [17-20]. Use of nonoperative alternatives is connected with increased morbidity and mortality [21]. Fibrinolysis is seldom a one-stage procedure [22]. A nationwide surveillance study from Germany revealed that a third of those initially treated with noninvasive 
therapy required secondary, invasive treatment [23] The randomized trials though seemingly proving equality in use of fibrinolytics compared to thoracoscopy in fact showed only increase of the effusion volume, which could be the effect of further irritation of the pleura as well $[24,25]$. What is also important, the majority of the studies on fibrinolysis do not distinguish between $2^{\text {nd }}$ and $3^{\text {rd }}$ phase empyema; hence we do not know which ones were cured in aforementioned trials. Moreover, spontaneous fibrinolysis is a well-known process [26]. Thus, we found fibrinolysis as supportive therapy in further complicated cases to dilute or loosen the dense and drain-blocking pus and used it in 21 cases and equally in phase 2 and 3 empyema.

A few case reports showed that thoracostomy with intrathoracic negative pressure therapy combined with endobronchial valve implantation could manage pleural empyema with broncho-pleural fistula [27], but in children this kind of treatment is technically complicated due to anatomical conditions (such as a narrow bronchial lumen).

We are aware of limitations of the study. They are small groups, which undermines the statistical comparisons; the long period of collecting the data could bias the results due to changes in the disease itself and clinical management (antibiotics, vaccination, increased recognition of the need of surgical management). The study relies on retrospective data and its focus is objective outcomes; thus the patient perspective is not known.

\section{Conclusions}

Based on the results of the present study, the thoracoscopic approach is safely feasible in the organized, $3^{\text {rd }}$ stage of pleural empyema in children, and should thus be considered as the preferred approach as long as complete pulmonary decortication is achieved, which is a condition for complete re-expansion of the lung.

Furthermore, although we did not succeed in finding strong support of the earlier referral for surgery, we strongly believe the post-operative stay and general course of the disease would be milder whenever surgery would not be delayed by prolonged conservative medical treatment attempts.

\section{Conflict of interest}

The authors declare no conflict of interest.

\section{References}

1. McCauley L, Dean N. Pneumonia and empyema: causal, casual or unknown. J Thorac Dis 2015; 7: 992-8.

2. Balfour-Lynn IM, Abrahamson E, Cohen G, et al. BTS guidelines for the management of pleural infection in children. Paediatric Pleural Diseases Subcommittee of the BTS Standards of Care Committee. Thorax 2005; 60 Suppl 1: i1-21.

3. Hamm H, Light RW. Parapneumonic effusion and empyema. Eur Respir J 1997; 10: 1150-6.

4. Reichert M, Pösentrup B, Hecker A, etal. Lung decortication in phase III pleural empyema by video-assisted thoracoscopic surgery (VATS)-results of a learning curve study. J Thorac Dis 2018; 10: 4311-20.

5. Melloni G, Carretta A, Ciriaco P, et al. Decortication for chronic parapneumonic empyema: results of a prospective study. World J Surg 2004; 28: 488-93.

6. Knebel R, Fraga JC, Amantea SL, Isolan PBS. Videothoracoscopic surgery before and after chest tube drainage for children with complicated parapneumonic effusion. J Pediatr 2018; 94: 140-5.

7. Singh AP, Shukla AK, Sharma P, Shukla J. Surgical management of stage III pediatric empyema thoracis. Lung India 2018; 35 : 209-14.

8. Kelly MM, Shadman KA, Edmonson MB. Treatment trends and outcomes in US hospital stays of children with empyema. Pediatr Infect Dis J 2014; 33: 431-6.

9. Stefani A, Aramini B, della Casa G, et al. Preoperative predictors of successful surgical treatment in the management of parapneumonic empyema. Ann Thorac Surg 2013; 96: 1812-9.

10. Chan DT, Sihoe AD, Chan S, et al. Surgical treatment for empyema thoracis: is videoassisted thoracic surgery "better" than thoracotomy? Thorac Surg 2013; 84: 225-31.

11. Chambers A, Routledge T, Dunning J, Scarci M. Is video-assisted thoracoscopic surgical decortication superior to open surgery in the management of adults with primary empyema? Interact Cardiovasc Thorac Surg 2010; 11: 171-7.

12. Subotic D, Lardinois D, Hojski A. Minimally invasive thoracic surgery for empyema. Breathe (Sheff) 2018; 14: 302-10.

13. Kumar A, Asaf BB, Lingaraju VC, et al. Thoracoscopic decortication of stage III tuberculous empyema is effective and safe in selected cases. Ann Thorac Surg 2017; 104: 1688-94.

14. Hajjar WM, Ahmed I, Al-Nassar SA, et al. Video-assisted thoracoscopic decortication for the management of late stage pleural empyema, is it feasible? Ann Thorac Med 2016; 11: 71-8.

15. Chung JH, Lee SH, Kim KT, et al. Optimal timing of thoracoscopic drainage and decortication for empyema. Thorac Surg 2014; 97: 224-9.

16. Scarci M, Abah U, Solli P, et al. EACTS expert consensus statement for surgical management of pleural empyema. Eur J Cardiothorac Surg 2015; 48: 642-53.

17. Aydoğan M, Aydoğan A, Ozcan A, et al. Intrapleural streptokinase treatment in children with empyema. Eur J Pediatr 2008; 167: 739-44.

18. Maskell NA, Davies CW, Nunn AJ, et al. U.K. Controlled trial of intrapleural streptokinase for pleural infection. First Multicenter Intrapleural Sepsis Trial (MIST1) Group. N Engl J Med 2005; 352: 865-74. 
19. Rahman NM, Maskell NA, West A, et al. Intrapleural use of tissue plasminogen activator and DNase in pleural infection. N Engl J Med 2011; 365: 518-26.

20. Semenkovich TR, Olsen MA, Puri V, et al. Current state of empyema management. Ann Thorac Surg 2018; 105: 1589-96.

21. Cekirdekci A, Köksel O, Göncü T, et al. Management of parapneumonic empyema in children. Asian Cardiovasc Thorac Ann 2000; 8: 137-40

22. Mathew JL, Soni V, Singh M, et al. Intrapleural streptokinase is effective and safe for children with multi-loculated empyema regardless of the time from disease onset. Acta Paediatr 2018; 107: 2165-71.

23. Segerer FJ, Seeger K, Maier A, et al. Therapy of 645 children with parapneumonic effusion and empyema - a German nationwide surveillance study. Pediatr Pulmonol 2017; 52: 540-7.

24. Thomson AH, Hull J, Kumar MR, et al. Randomised trial of in trapleural urokinase in the treatment of childhood empyema. Thorax 2002; 57: 343-7.

25. St Peter SD, Tsao K, Spilde TL, et al. Thoracoscopic decortication vs tube thoracostomy with fibrinolysis for empyema in children: a prospective, randomized trial. J Pediatr Surg 2009; 44: 106-11.

26. Kho P, Karunanantham J, Leung M, Lim E. Debridement alone without decortication can achieve lung re-expansion in patients with empyema: an observational study. Interact Cardiovasc Thorac Surg 2011; 12: 724-7.

27. Panko S, Vakulich D, Karpitski A, et al. Intrathoracic negative pressure therapy and/or endobronchial valve for pleural empyema minimal invasive management: case series of thirteen patients and review of the literature. Videosurgery Miniinv https://doi.org/10.5114/wiitm.2020.93210.

Received: 12.04 .2020 , accepted: 30.04 .2020 